Cad.Est.Ling., Campinas, 52(1): 37-44, Jan./Jun. 2010

\title{
¿ESPOSIBLE LA NOCIÓN DE ENUNCIACIÓN EN LA LINGÜÚSTICA SAUSSUREANA? REFLEXIONES SOBRE EL LUGAR DEL SUJETO
}

\author{
CECILIA BLEZIODUCRET \\ (UNIVERSIDADDELAREPÚBLICA, URUGUAY)
}

\begin{abstract}
RESUMEN: En la lingüística que se considera "saussureana" hay una imposibilidad teórica de considerar el sujeto, que, no obstante, parece insistir como problemática. Dentro de la teoría lingüística, lato sensu, podría considerarse la noción de "enunciación" como un posible abordaje de la cuestión del sujeto. En este artículo, me referiré especialmente a la noción de enunciación de Benveniste (1970) y Ducrot (principalmente sus trabajos de la década de 1980). Así, ¿podrían postularse afectaciones entre esta noción y una lingüística que pueda llamarse saussureana? Concretamente, en el Curso de lingüística general y en los Escritos sobre lingüística general de de Saussure la noción de enunciación no aparece explícitamente abordada, no obstante puede rastrearse elementos que se vinculan con ella. Aun así, la enunciación no parece ser suficiente para abordar la cuestión del sujeto que, según otras lecturas, aparece en el Curso; esta conceptualización que quedaría mejor planteada dentro de los parámetros teóricos de la lingüisterie más que de la lingüística.
\end{abstract}

\begin{abstract}
In the Linguistics that is considered to be "Saussurean" there is a theoretical impossibility to consider the subject, who, although, seems to insist as a problem. In the Linguistics theory, lato sensu, the notion of "enunciation" could be considered as a possible approach to the matter of the subject. In this article, I referred specially to the notion of enunciation of Benveniste (1970) and Ducrot (mainly his words from the decade of 1980). In this sense, could we postulate there is a relationship between this notion and a so called Saussearean Linguistics? In a concrete manner, in the General Linguistics Course and in the Writings on general Linguistics of de Saussure the notion of enunciation does not appear in an explicit approach, in spite of the fact that there are some elements in connection with it that we can follow. Even though, the issue of enunciation does not seem to be enough to focus on the matter of the subject, that, according to other readings, appears in the Course; this conceptualisation that could be better developed in the theoretical parameters of the lingüisterie than in the Linguistics.
\end{abstract}

\section{CONSIDERACIONESGENERALES}

Este trabajo se enmarca en la Línea de Investigación Estudio de lo Didáctico como Acontecimiento Discursivo e Intersubjetividad (EDADI), dentro del Departamento de Psicología de la Educación y Didáctica de la Facultad de Humanidades y Ciencias de la Educación de la UdelaR; en particular, se enmarca dentro de las reflexiones del grupo de trabajo en Lengua/Lenguaje y Enseñanza, ambos dirigidos por Luis E. Behares. La subjetividad y de la intersubjetividad han sido preguntas recurrentes, tematizadas en indagaciones previas (por ejemplo, Blezio, 2005a y 2005b). En este trabajo procuré buscar en la lingüística saussureana qué elementos hay para profundizar estas cuestiones. Así es que trabajaré fundamentalmente la noción de "enunciación", donde parece tratarse la subjetividad. 
En las lecturas tradicionales de de Saussure -y del estructuralismo en general- se ha dicho que el sujeto queda excluido (ver Henry, 1977; Milner, 1978; Pêcheux y Gadet, 1981) . A partir de ahí se funda toda una lingüística que, dadas las coordenadas en las que se sitúa, hace imposible plantear la noción de sujeto. Pero el sujeto retorna, de diversas maneras; en lingüística, a través de sus expresiones más "aplicadas": psicolingüística, sociolingüística, pragmática. Más contemporáneamente -y más vinculado con mi filiación teórica-se puede pensar en otro sujeto, tomado del psicoanálisis: el del inconsciente, el del lenguaje. Este sujeto ya no es dueño de su decir sino que es hablado; es el de lalangue. Este sujeto se reconoce en otras lecturas del Curso de linguiística general (de Saussure, 1916), como las iniciadas por Pêcheux, Lacan, Milner y otros, que parecen confirmarse en los Escritos sobre lingüística general (de Saussure, 2002).

La tradición lingüística ha hecho intentos de formular teóricamente esta insistencia del sujeto. Uno que merece particular interés es a partir de la noción de "enunciación" de la tradición teórica francesa y sus consecuencias teóricas, por ejemplo, la noción de la polifonía, la teoría de la argumentación en la lengua. Entonces, ¿qué cruces es posible postular entre la noción de enunciación y una lingüística que pueda llamarse "saussureana”?

\section{BREVEMENTE,LA NOCIÓNDEENUNCIACIÓNENLINGÜÍSTICA}

Esta noción se ubica en la tradición filosófica y retórica, y tuvo desarrollos en la época medieval. Ingresa a la una cierta tradición lógico-filosófico-lingüística a partir de Port Royal, y luego fue retomada por autores pos Curso, como Benveniste y Ducrot ${ }^{1}$.

Émile Benveniste refiere a este aspecto en algunos de sus capítulos de sus Problèmes de linguistique générale. Por ejemplo, hay un capítulo denominado "El aparato formal de la enunciación"2 (en el tomo dos, en el apartado dedicado a la comunicación) (Benveniste, 1974: 79-88). Este capítulo comienza con una referencia a la sintaxis, como el conjunto de reglas que fijan, regulan, el "emploi des formes". Sin embargo, el autor establece una diferencia entre "conditions de emploi des formes" y "conditions de emploi de la langue": "Ce sont en realité des mondes différents, et il peut éter util d'insister sur cette différence, qui implique une autre manière de voir les mêmes choses, une autre manière de les décrire et de les interpréter" (Benveniste, 1974: 79). Ubicado en las condiciones de uso de la lengua, define la noción de enunciación como la "mise en fonctionnement de la langue par un acte individuel d'utilisation" (Benveniste, 1974: 80) y, como tal, tiene una condición específica: es “l'acte même de produire un énoncé et non le texte del'énoncé” (Benveniste, 1974: 80).

Así, la enunciación supone "la conversion individuelle de la langue en discours" (Benveniste, 1974: 81).

${ }^{1}$ También Bajtín, aunque más en los márgenes de la lingüística, por lo que no será considerado en este trabajo.

${ }^{2}$ En esta obra hay también otros artículos que refieren a la enunciación, por ejemplo: "De la subjecitivité dans le langage" (Benveniste, 1966: 258-266) o "L'antonyme et le pronom en français moderne (Benveniste, 1974: 197-214) que, no obstante, no serán abordados en este trabajo. 
Hay un locutor - primera condición necesaria para que haya enunciación-que "mobilise la langue pour son compte" (Benveniste, 1974: 81), la toma como instrumento: "l'énonciation peut se définir, par rapport à la langue, comme un procès d'appropriation" (Benveniste, 1974: 82).

Benveniste sostiene:

“Avant la énonciation, la langue n'est que la posibilité de la langue. Après l'énonciation, la langue est effectuée en une instance de discours, qui émane d'un locuteur, forme sonore qui atteint un auditeur et qui suscite autre énonciation en retour". (Benveniste, 1974: 81-82).

La enunciación presenta caracteres lingüísticos: implica al menos dos (locutor y alocutario) -lo cual se hace evidente con las marcas personales de primera y segunda persona así como con las marcas de ostensión (Benveniste, 1974: 81-82) y aporta las condiciones necesarias para las grandes funciones sintácticas (interrogación, orden, aserción; queda fuera la negación en tanto que operación lógica) (Benveniste, 1974: 84).

En la enunciación, la lengua se encuentra empleada en una cierta relación con el mundo; el locutor se apropia de la lengua y la condición misma de esta apropiación es la necesidad de referir, por el discurso, y la posibilidad de correferir idénticamente, en el consenso pragmático que hace de cada locutor un colocutor. Esta referencia es parte integrante de la enunciación (Benveniste, 1974: 82).

Así, se destaca la importancia de la comunicación:

“ce qui en générale caractérise l'énonciation est l'accentuation de la relation discoursive au partenarire, que celui-ci sois réel ou imaginé, individuel ou collectif. Cette caractéristique posse par nécessité ce qu'on peut appeler le cadre figuratif de l'énonciation. Comme forme de discours, l'énontiaton pose deux 'figures' également nécessaires, l'une source, l'autre bur de la énonciation. C'est la structure du dialogue” (Benveniste, 1974: 85).

El sujeto que parece estar definido aquí es un sujeto capaz de "utilizar la lengua" como instrumento, en el marco de una relación enunciativa y del diálogo.

La noción de "enunciación" también fue formulada por Oswald Ducrot; este autor tiene una extensa obra y el concepto tuvo matices diferentes; a los efectos de este trabajo, se considerarán fundamentalmente sus textos de la década de 1980.

Ducrot cuestiona la unicidad del sujeto hablante y tematiza la noción de enunciación a partir de la polifonía y de su tratamiento de la presuposición; a la vez, asumir la noción de enunciación tiene consecuencias, por ejemplo, para su teoría de la argumentación en la lengua (sostenida conjuntamente con Anscombre, Carell, entre otros ${ }^{3}$ ). Define enunciación como "acontecimiento constituido por la aparición de un enunciado" (Ducrot, 1984: 183).

En ese marco, opone los conceptos de oración (entidad lingüística abstracta y puramente teórica formada por un conjunto de palabras combinadas según reglas de la sintaxis, que se sitúa fuera de toda situación de discurso) y el de enunciado (es decir,

${ }^{3}$ El autor reconoce la importancia del componente argumentativo, que concibe de orden puramente lingüístico. Esto le permite incluir en la descripción lingüística ciertos fenómenos que de otro modo no tendrían explicación (por ejemplo, el hecho de que dos expresiones contradictorias puedan tener una misma orientación argumentativa). El componente informativo de la frase queda subsumido e incluso los usos informativos se derivan de un componente más profundo que es, en última instancia, argumentativo. 
aquello que produce un locutor y que entiende un auditor, que constituye una ocurrencia particular de una oración (Ducrot et al., 1980: 7). El valor semántico de la oración es la “significación". Su atribución no proviene de la observación sino de la explicación: se trata de asignar una significación que permita prever, a partir de ella, el sentido que tendrá su enunciado en cada situación de uso (Ducrot et al., 1980: 8); pero no se trata del "sentido literal" (como elemento semántico mínimo contenido en el sentido de todos los enunciados de una oración) (Ducrot et al., 1980: 11). Tampoco es un componente del sentido del enunciado. La significación se caracteriza por contener instrucciones para quienes deberán interpretar el enunciado, para quienes deben buscar información en la situación de discurso y utilizarla para reconstruir el sentido presentado por el locutor. La significación sólo se comprende cabalmente incluyendo la dimensión de los enunciados (Ducrot et al., 1980: 12).

La enunciación tiene un rol fundamental y constitutivo en la descripción lingüística; no se suma al sentido literal modificándolo, completándolo o atenuándolo (Ducrot, 1980: 9). Implica que el "sentido" de los enunciados es diferente según la situación de discurso, es decir, hay múltiples lecturas posibles para un enunciado. En una situación de discurso aparecen todas las creencias y las intenciones de los locutores; según las que se toman en cuenta y las que se dejan de lado para interpretar un enunciado, varía la interpretación (Ducrot et al., 1980: 17-18).

Ducrot sostiene que el sentido del enunciado -incluso cuando se entiende este término en su acepción más estricta, más gramatical- no puede ser descrito sin hacer referencia a algunas intenciones de enunciación: es imposible aislar una parte cualquiera del sentido que no esté marcada por la función enunciativa (Ducrot, 1980: 8-9). Ducrot lo concibe no como "la suma de la significación más otra cosa, sino como una construcción que, habida cuenta de la situación de discurso, se opera a partir de las consignas especificadas en la significación" (Ducrot, 1984: 186). Todo enunciado aporta una cualificación de su enunciación, que constituye el sentido del enunciado (Ducrot, 1984: 177-178); éste-el sentido- resulta entonces una descripción de la enunciación (Ducrot, 1984: 187).

El enunciado no es definible en términos extralingüísticos y no es explicado posteriormente por la actividad del habla. Esta perspectiva apoya la tesis de la especificidad absoluta del orden lingüístico: "hay una primacía de la lengua, siendo ésta irreductible a todo lo que no sea ella misma" (Ducrot, 1984: 67).

Para Ducrot: "una entidad ligüística toma toda su realidad del discurso donde tiene lugar -no del que está empíricamente incorporada sino del que exige, el que reivindica. Es esta reivindicación lo que la constituye. (Ducrot, 1980: 11). El objeto teórico "lengua" no puede ser construido si no se hace alusión a la actividad de habla. Esta inclusión de la enunciación no implica considerarla en lo que tiene de histórico o contingente sino dentro del universo formal de la lengua. No aparece la noción de un sujeto que realiza un acto de habla. La enunciación se incluye en el enunciado: el valor semántico de un enunciado no puede definirse sino en relación con el uso (y esto también se apoya en su tratamiento de la presuposición). Además, el propio Ducrot, en su discusión con Henry menciona la ignorancia del locutor sobre lo que sus propias palabras significan (e incluso habla de "lapsus presuposicionales", aunque les da un carácter excepcional) (Ducrot, 1977: 206-207).

El sujeto parece en una relación de dominio menor respecto de su lengua, no obstante este aspecto no es trabajado por Ducrot y sólo puede inferirse, sin referencias explícitas. 


\section{INDICIOS DE LA ENUNCIACIÓN ENEL CURSO Y LOS ESCRITOS}

En estos textos de de Saussure la enunciación no está trabajada explícitamente, en un ejercicio de visualizar cuáles serían los posibles agujeros por donde establecer cierta relación con ella. Tal vez algunas pistas puedan ubicarse en la distinción lengua/habla, en la linealidad del significante y, por ella, su capacidad para formar cadenas (CLG: 133-134), en las relaciones sintagmáticas y aspectos más generales en torno a la comunicación.

A partir de la distinción lengua/habla parece quedar claro que en la lingüística saussureana la cuestión del sujeto queda excluida: la lengua se formula como sistema y todo lo singular queda del lado del habla (que sería, por tanto, el lugar para el sujeto).

Al ubicar la lengua en los hechos del lenguaje (CLG: 54), en el Curso se propone: "hay que situarse ante el acto individual que permite reconstruir el circuito de la palabra. Este acto supone por lo menos dos individuos: es el minimum exigible para que el circuito sea completo." (CLG: 54). Se trata de un circuito de comunicación, que hace referencia a lo discursivo y supone al hablante. Así:

"La lengua no es una función del sujeto hablante, es el producto que el individuo registra
pasivamente; nunca supone premeditación, y la reflexión no interviene en ella más que para
la actividad de clasificar [...] El habla es, por el contrario, un acto individual de voluntad y de
inteligencia, en el cual conviene distinguir: $1^{\circ}$, las combinaciones por las que el sujeto hablante
utiliza el código de la lengua con miras a expresar su pensamiento personal; $2^{\circ}$, el mecanismo
psicofísico que le permita exteriorizar esas combinaciones."(CLG: 57 ).

La materialidad de la lengua se asienta en cada hablante y en todos a la vez, pero fuera de su voluntad.

El Curso establece que la oración es una unidad del habla, sin embargo no así el sintagma (CLG: 209). A la vez, en virtud del encadenamiento "las palabras contraen entre sí relaciones fundadas en el carácter lineal de la lengua, que excluye la posibilidad de pronunciar dos elementos a la vez"; y esta relación in praesentia se da en el discurso (CLG: 207). En los Escritos, en un capítulo breve, "Nota sobre el discurso" (ELG: 245) se plantea:

"La lengua sólo se ha creado para el discurso, pero ¿qué separa el discurso de la lengua, o qué es lo que en un determinado momento permite decir que la lengua entra en acción como discurso? [...] La serie de palabras, por rica que sea, por más ideas que evoque, jamás indicará a un individuo humano que otro individuo, al pronunciarlas, quiera significarle algo. [...] el discurso consiste, aunque sea rudimentariamente y por vías que ignoramos, en afirmar un lazo entre dos de los conceptos que se presentan revestidos de forma lingüística, mientras que la lengua, previamente, sólo realiza conceptos aislados, que quedan en espera de ser relacionados entre ellos para que haya significación de pensamiento." (ELG: 245).

No obstante la preeminencia del principio del valor para explicar los fenómenos lingüísticos, hay una dimensión discursiva:

"Mientras que es necesario un análisis para establecer los elementos de la palabra, la propia palabra no resulta del análisis de la frase. Pues la frase no existe más que en el habla, en la lengua discursiva, mientras que la palabra es una unidad que vive fuera de todo discurso en el tesoro mental." (ELG: 112). 


\section{REFLEXIONESFINALES}

La teoría de la enunciación ${ }^{4}$ así como la de los actos de habla parecen ser, en última instancia, "lingüísticas del habla"': no tratan de un sujeto que sea parte de la lengua sino de un sujeto del habla. Postular que algo escapa al control de los hablantes (como en el planteo de Ducrot) no es suficiente para no estar en el ámbito del sujeto psicológico.

El concepto de "habla" es diferente del de "enunciación" (de hecho, es una pregunta del propio Benveniste); habría que explorar más las relaciones entre ambas.

Parece quedar de manifiesto que la noción de enunciación no es saussureana, no sólo porque de Saussure no la incluya en sus trabajos sino porque no tiene cabida dentro de su perspectiva. Esta discusión podría enmarcarse en otra más general, acerca de los conceptos de sujeto que manejan ambas lingüísticas. Ni para el Curso y los Escritos ni para la teoría de la enunciación parece importante detenerse en este concepto ${ }^{6}$. Así, en los Escritos:

"La conquista de estos últimos años es haber situado por fin no sólo todo lo que es el lenguaje y la lengua en su verdadero hogar, el sujeto hablante, sino haber considerado a éste como ser humano y como ser social." (ELG: 120).

Frecuentemente, utiliza términos como “conciencia" (ELG: 41, 49, 52, 167, 172) o "mente" (ELG: 43, 47). Pero a la vez, y ya desde el Curso, en la comparación de entre la lengua y el juego de ajedrez, de Saussure sostiene: "Para que la partida de ajedrez se pareciera en todo a la legua sería necesario suponer un jugador inconsciente o ininteligente.” (CLG: 160), lo cual apunta a otra dimensión. Esta dimensión también aparece esbozada en los Escritos:

\footnotetext{
"Todas las modificaciones, sean fonéticas o gramaticales (analógicas), tienen lugar exclusivamente en lo discursivo. En ningún momento el individuo somete a revisión el tesoro mental de la lengua que tiene en sí ni crea con la cabeza fría formas nuevas (por ejemplo, tranquilamente [ ]) que se propone (promete) 'colocar' en su próximo discurso. Toda innovación ocurre de modo improvisado, hablando, y de ahí penetra o en el tesoro íntimo del oyente o en el del orador; pero entonces se produce a propósito del lenguaje discursivo." (ELG: 95).
}

Lecturas posteriores de los Escritos ${ }^{7}$ postulan que hay un sujeto en de Saussure, que, no obstante, tampoco parecería tocarse con la noción de enunciación. Pero éste es un aspecto todavía a explorar.

\footnotetext{
${ }^{4}$ Lato sensu: en rigor, no se trata de una teoría en sentido epistemológico estricto.
}

${ }^{5}$ La lingüística del habla fue planteada como posibilidad por de Saussure en el Curso, pero sólo mencionada, bajo riesgo de confundirla con la lingüística propiamente dicha, cuyo objeto es la lengua (CLG: 65-66).

${ }^{6}$ Aunque puede decirse que lo que se pone de manifiesto es que por más que se evite esta noción, siempre vuelve de alguna forma u otra.

${ }^{7}$ Por ejemplo, Maniglier (2006). Este texto fue trabajado por Marie-Claude Thomas (2007). 
A pesar de que las dos corrientes trabajan con la idea de sistema, se trata de dos conceptos diferentes: en de Saussure se trata de un sistema de oposiciones o valores puros $^{8}$; en Benveniste o Ducrot, se trata de un funcionamiento en el uso. En el primer caso, "formal" puede leerse como matematizado; en cambio en el segundo, lo formal parece más vinculado a una cierta condición de abstracto.

Por otra parte, la enunciación -la oración, la cadena hablada, la dimensión sintagmática, tanto se trate de un punto de vista más apegado a la gramática o a las reglas de uso-implican la idea del sentido que presupone, en última instancia, la transparencia de la comunicación; es decir, desde esa perspectiva no se toma la oposición sentido-sinsentido; otra perspectiva diferente es la que marca el significante (ver Lacan, 1955-1956 y 1974). De Saussure puede leerse como teoría del signo o del valor, pero no de la oración o la cadena hablada. El funcionamiento del sistema para el Curso es la puesta en marcha del principio del valor, incluso en el plano de la sintaxis. En los Escritos, se afirma:

\footnotetext{
"una regla de 'sintaxis' que determine en qué casos se utiliza el pretérito perfecto -o una regla 'morfológica' (en sentido estricto) que determine cuál es la forma del pretérito perfecto; o una regla que pretenda ser 'fonética' y que determine en qué caso se elide una vocal o en qué caso una /pi/ es sustituida por una /psi/- pertenece, mediante una profunda e irreductible unión, al MISMO ORDEN DE HECHOS: conocer el juego de los signos por medio de sus diferencias en un momento dado." (ELG: 39)
}

Una lectura más en profundidad de los Escritos, todavía pendiente, arrojaría mayor luz sobre las cuestiones abordadas en este trabajo. Así, este trabajo no es más que un punto en el tratamiento de una pregunta que insiste. Tal vez esta pregunta no pueda avanzar dentro de la lingüística sino en la lingüistería.

\section{REFERENCIASBIBLIOGRÁFICAS}

BENVENISTE, Émile Problèmes de linguistique générale 1, Gallimard, Paris, 1966.

.L'appareil formel de l'énonciation. In: Problèmes de linguistique générale 2, Gallimard, Paris, 1974, pp. 79-88.

BLEZIO, Cecilia. Intersubjetividad y resignificación. In: BEHARES L. E. \& S. COLOMBO (Comps.) Enseñanza del saber - saber de la enseñanza, FHCE, Montevideo, 2005a. pp. 61-66.

.Reflexiones sobre el sujeto en el acontecimiento didáctico. Conversación, diciembre de 2005, Montevideo, 2005b. pp. 19-26.

DE SAUSSURE, Ferdinand. (1916) Curso de lingüística general, Losada, Buenos Aires, 1967. .(2002) Escritos sobre lingüística general, Gedisa, Barcelona, 2004.

DUCROT, Oswald. (1974) Nota sobre a pressuposição e o sentido literal. In: HENRY, P. A ferramenta imperfeita, Unicamp, Campinas, 1992, pp. 203-238.

.Les échelles argumentatives, Éditions du Minuit, Paris, 1980.

(1984) El decir y lo dicho. Polifonía de la enunciación, Paidós, Barcelona, 1986. .et al. Les mots du discours, Éditions du Minuit, Paris, 1980.

\footnotetext{
${ }^{8}$ Aunque también se podría admitir el énfasis en el "sistema de signos".
} 
DUCRET - ¿Es possible la noción de enunciación en la linguüística...

GADET, Françoise \& Michel PÊCHEUX (1981) La lengua de nunca acabar, FCE, México, 1984.

HENRY, Paul. (1977) A ferramenta imperfeita, Unicamp, Campinas, 1992.

LACAN, Jacques. (1955-1956) El Seminario. Libro 3. Las Psicosis, Paidós, Buenos Aires, 2002. .(1972-1973) El Seminario. Libro 20. Aun, Paidós, Buenos Aires, 1995.

MANIGLIER, Patrice La vie énigmatique des signes. Saussure et la naissace du structuralisme, Éditions Léo Scheer, Paris, 2006.

MILNER, Jean-Claude. L'Amour de la Langue. Paris: Du Seuil. 1978.

THOMAS, Marie-Claude. (2007) Sensaciones de lengua. A propósito de La vie énigmatique des signes. Saussure et la naissance du structuralisme, de Patrice Maniglier. Litoral. Inquietante extrañeza, $\mathrm{N}^{\circ}$ 40, EDELP, Córdoba, pp. 61-91. 